\title{
Wigner's Theorem on Symmetries in Indefinite Metric Spaces*
}

\author{
L. Bracci ${ }^{\star}$, G. Morchio ${ }^{\star \star}$, and F. Strocchi \\ Scuola Normale Superiore, Pisa, Italy \\ Received October 21, 1974
}

\begin{abstract}
The description of symmetries in indefinite metric spaces is investigated. It is shown that ray transformations preserving the modulus of an indefinite scalar product can be implemented by linear or antilinear vector transformations which are generalized unitary or antiunitary operators with respect to the indefinite scalar product. A number of interesting features arise since such operators need not to be bounded.
\end{abstract}

\section{Introduction}

Wigner's theorem $[1,2]$ on ray transformations in Hilbert spaces plays a fundamental rôle in the foundations of quantum mechanics $[3,4]$ and it has deep connections with the mathematical theory of projective spaces [5-7]. In the proof of the theorem a crucial rôle is played by the Hilbert space structure and, in particular, by the positivity of the scalar product. On the other hand it has become more and more evident that indefinite metric spaces may be more useful both for the discussion of physical problems as well as for more genuine mathematical questions. As far as the physical applications are concerned the growing evidence comes mainly from the theory of quantum fields for which the use of an indefinite metric has been advocated several times in the past [8-10] as a solution of the divergence problem in quantum field theory $[11,12]$ and as a method to obtain better regularity properties for theories previously regarded as untractable $[13-16]$. For several physically interesting theories the use of an indefinite metric is not only a promising suggestion but an unavoidable feature if one wants to preserve some basic properties of the fields like relativistic covariance and locality [17-19].

From a mathematical point of view the interest of indefinite metric spaces has been pioneered by the Russian mathematicians [20-22] and we refer to their papers for general motivations as well as for an exposition of the results obtained in that field. It may be interesting to stress that indefinite metric spaces appear very useful also in solving stability problems in the classical theory of damped oscillations and in general as a powerful tool for solving systems of differential equations [24]. In particular canonical linear differential equations with a periodic Hamiltonian have been studied with success using indefinite metric spaces [25].

In the following by an indefinite metric space we mean an Hilbert space $H$ equipped with a bounded symmetric sesquilinear form $\langle\cdot, \cdot\rangle=(\cdot, \eta \cdot)$, where $(\cdot, \cdot)$

$\star$ Research partially supported by INFN.

$\star \star$ Present address: Istituto di Fisica dell'Università, Pisa.

$\star \star \star$ Present address: Istituto di Fisica dell'Università, Torino. 
is the ordinary scalar product in $H$ and $\eta$ has a bounded inverse. In the physical as well as in the mathematical applications mentioned above the basic quantity of the theory is the product $\langle\cdot, \cdot\rangle$ rather than $(\cdot, \cdot)$. For example it is in terms of $\langle\cdot, \cdot\rangle$ that one computes all the physical quantities of the theory or one discusses the mathematical properties of a given differential operator.

It is therefore natural to ask the question analogue to the statement of Wigner's theorem: given a ray transformation between rays of the Hilbert space $H$, preserving the modulus of the product $\langle\cdot, \cdot\rangle$, is it possible to implement such a transformation by a linear or antilinear vector transformation. The answer to this question is crucial for the foundations of a quantum mechanical description of symmetries in indefinite metric spaces as well as for the existence of semigroups associated to time evolution, space translation etc.

As it will be clear in the following there are fundamental differences with respect to the Wigner's case, mainly because vector transformations preserving the indefinite scalar product need not to be bounded and therefore non trivial domain question arise. Another delicate point is the linear structure of the domain on which the transformation is defined: as it will appear in the following it plays a crucial rôle.

Before stating and proving the theorem it is necessary to give some basic definitions and discuss some crucial features of vector transformations preserving the indefinite metric ( $\eta$-unitary operators) (Section 2 ). The hypotheses of the theorem and their relevance will be stated and discussed in Section 3. The proof will be given in Section 4.

\section{Unitary Operators in Indefinite Metric Spaces and Their Basic Properties}

Definition 1. By an indefinite metric space we mean a complex (separable) Hilbert space $H$ equipped with a bounded, hermitian and not degenerate sesquilinear form $\langle\cdot, \cdot\rangle=(\cdot, \eta \cdot),(\cdot, \cdot)$ being the ordinary scalar product in $H$, such that $\eta$ is a self-adjoint operator with bounded inverse.

Proposition 1. Without loss of generality an indefinite metric space can be assumed to have a metric operator with the property $\eta^{2}=1$.

Unless explicitely stated, we will in the following assume that an indefinite metric space has $\eta^{2}=1$.

Definition 2. An operator $U$ with $\bar{D}_{U}=H=\bar{\Delta}_{U}$ and such that

$$
\langle U x, U y\rangle=\langle x, y\rangle, \quad \forall x, y \in D_{U}
$$

is called a unitary operator in the indefinite metric space or briefly a $\eta$-unitary operator.

In the physical applications Eq. (1) is usually the most one is allowed to require since it may be difficult to justify additional properties of $U$ like linearity, existence of the inverse and/or its boundedness on the basis of purely physical considerations. For example in quantum field theory the invariance of the Wightman functions under a given mapping $U$, yields the validity of Eq. (1) for the dense domain obtained by applying local fields to the vacuum. The extension of the mapping to every vector of $H$ is often not possible. From a technical point of view, since $U$ is 
usually an unbounded operator, the closure properties of $U$ are often useful. For these reasons it is of some interest to state the following

Proposition 2. A $\eta$-unitary operator is a linear operator, it has an inverse which is a $\eta$-unitary operator and it is closable.

Proof. We start by proving that $U$ has an inverse, i.e. that $U x=U y$ implies $x=y$. In fact, for any $z \in D_{U}$

$$
0=\langle U x-U y, U z\rangle=\langle x, z\rangle-\langle y, z\rangle=\langle x-y, z\rangle=(x-y, \eta z)
$$

and since $\overline{\eta D_{U}}=H$, one has $x=y$. Thus $U^{-1}$ exists and $D_{U^{-1}}=\Delta_{U}, \Delta_{U^{-1}}=D_{U}$. Moreover, $\forall x, y \in D_{U^{-1}}$

$$
\langle x, y\rangle=\left\langle U U^{-1} x, U U^{-1} y\right\rangle=\left\langle U^{-1} x, U^{-1} y\right\rangle
$$

i.e. $U^{-1}$ is a $\eta$-unitary operator. Finally $\forall x, y \in D_{U}, z \in \Delta_{U}$

$$
\begin{aligned}
\langle U(\alpha x+\beta y), z\rangle & =\left\langle\alpha x+\beta y, z^{\prime}\right\rangle=\alpha\left\langle x, z^{\prime}\right\rangle+\beta\left\langle y, z^{\prime}\right\rangle \\
& =\alpha\langle U x, z\rangle+\beta\langle U y, z\rangle
\end{aligned}
$$

i.e.

which implies

$$
\langle U(\alpha x+\beta y)-\alpha U x-\beta U y, z\rangle=0
$$

$$
U(\alpha x+\beta y)=\alpha U x+\beta U y .
$$

To prove that $U$ is closable one first notices that $\forall x \in D_{U}, y \in \eta D_{U^{-1}}, y=\eta z$

$$
(U x, y)=\langle U x, z\rangle=\left\langle x, U^{-1} z\right\rangle=\left(x, \eta U^{-1} \eta y\right) .
$$

Thus $U^{*}$ exists, $\eta U^{-1} \eta \subset U^{*}$ and $\eta D_{U^{-1}} \subset D_{U^{*}}$. Hence $\bar{D}_{U^{*}}=H, U^{* *}$ exists and $U \subset U^{* *}$ is closable.

Remark. The occurrence of an indefinite metric operator in Eq. (1) does not allow to conclude, as in the positive metric case, that $U$ is bounded. This is a fundamental difference which makes $\eta$-unitary operators much more difficult to treat than unitary operators. For these reasons, in the mathematical literature on indefinite metric spaces [21-24], the boundedness property is required in the definition of $\eta$-unitary operators. However, since this property is in general not shared by operators occurring in the physical applications we prefer to omit it in the Definition 2.

Definition 3. An operator $V$ with $\bar{D}_{V}=H=\bar{\Delta}_{V}$ and such that

$$
\langle V x, V y\rangle=\langle y, x\rangle, \quad \forall x, y \in D_{V}
$$

is called a $\eta$-antiunitary operator.

Proposition 3. A $\eta$-antiunitary operator $V$ can be written as a product $V=K U$, where $K$ is the complex conjugation operator (in a suitable basis such that $\eta K=K \eta$ ), $K^{2}=1$, and $U$ is a $\eta$ unitary operator.

A $\eta$-antiunitary operator is antilinear, it has an inverse which is a $\eta$-antiunitary operator and it is closable.

Proof. To prove the first part of Proposition 3 it suffices to note that $U \equiv K V$ is a $\eta$-unitary operator and therefore $K U=K^{2} V=V$. The second part of Proposi- 
tion 3 follows easily from Proposition 2 since $K$ is a bounded antilinear operator with $K^{-1}=K$.

Just as antiunitary operators arise when one goes from real to complex Hilbert spaces, a new class of operators, closely related to unitary operators, naturally occurs when the "scalar" product is allowed to be indefinite.

Definition 4. An operator $U$ with $\bar{D}_{U}=H=\bar{\Delta}_{U}$ and such that

$$
\langle U x, U y\rangle=-\langle x, y\rangle, \quad \forall x, y \in D_{U}
$$

is called a $\eta$-pseudounitary operator. In a similar way one defines $\eta$-pseudo antiunitary operators.

The occurrence of $\eta$-pseudounitary operators is tightly bound to the indefinite metric, since $\langle U x, U x\rangle=-\langle x, x\rangle$ would otherwise be untenable. It is important to stress that $\eta$-pseudounitary or $\eta$-pseudoantiunitary operators may exist only in indefinite metric spaces in which the eigenvalues +1 and -1 of the metric operator $\eta$ have the same multiplicity. They cannot exist if one multiplicity is finite and the other infinite or if they are both finite but unequal.

A special subclass of $\eta$-pseudounitary operators are those operators $C$ which are bounded, self adjoint and satisfy $C^{2}=1$. They satisfy

$$
C \eta C=-\eta
$$

and they will be called $\eta$-reflecting operators. It is not difficult to see that a $\eta$ pseudounitary operator $V$ can be written as a product $V=C U$ where $C$ is a $\eta$-reflecting operator and $U$ is a $\eta$-unitary operator. Similar statements hold for $\eta$-pseudoantiunitary operators.

Proposition 4. $A \eta$-pseudounitary ( $\eta$-pseudoantiunitary) operator is a linear (antilinear) operator, it has an inverse which is a $\eta$-pseudounitary ( $\eta$-pseudoantiunitary) operator and it is closable.

\section{Ray Transformations in Indefinite Metric Spaces}

Definition 5. Given a vector $x \in H$, the set of vectors of the form $\lambda x$, with $|\lambda|=1, \lambda \in C$, is called the ray associated to $x$ and it will be denoted by $\underline{x}$. The vector $x$ is said to belong to $\underline{x}: x \in \underline{x}$.

Clearly if $y=\mu x,|\mu|=1$, one has $\underline{y}=\underline{x}$. For the rays of an indefinite metric space, one defines an ordinary scalar product

$$
\underline{x} \cdot \underline{y} \equiv|(x, y)| \quad x \in \underline{x}, y \in \underline{y}
$$

also denoted by $|(\underline{x}, \underline{y})|$, and a semidefinite product

$$
\underline{x}_{\dot{\eta}} \underline{y}=\underline{x} \cdot \eta \underline{y} \equiv|\langle x, y\rangle| \quad x \in \underline{x}, y \in \underline{y}
$$

also denoted by $|\langle\underline{x}, \underline{y}\rangle| \equiv|(\underline{x}, \eta \underline{y})|$.

In contrast with the Wigner case, some technical difficulty arises because one cannot normalize the rays with respect to the indefinite scalar product. It may be relevant to note that by the non degeneracy of the metric operator if a ray $\underline{x}$ satisfies $\underline{x} \dot{y} \underline{y}=0, \forall \underline{y}$, then $\underline{x}$ is the ray $\underline{0}$ associated to the zero vector.

Definition 6. A ray transformation $T$ is a mapping $T: \underline{x} \rightarrow \underline{x^{\prime}} \equiv T \underline{x}$ of rays into rays. 
A ray transformation $T$ is induced by a linear operator $\mathscr{T}$ in $H$ if $T: \underline{x} \rightarrow T \underline{x}$ $=\underline{T} x$; in this case we will denote the ray transformation and the linear operator by the same letter, for simplicity.

A special kind of ray transformations are those which preserve the semidefinite scalar product between rays

$$
T \underline{x} \cdot \eta T \underline{y}=|\langle T \underline{x}, T \underline{y}\rangle|=|\langle\underline{x}, \underline{y}\rangle|
$$

and they are candidates to describe symmetry operations in indefinite metric spaces.

In general a symmetry operation is not defined for all the rays of $H$. It is reasonable however to require that a symmetry operation $T$ is invertible, defined on a set of rays associated to a dense linear manifold $D$ of $H$, and that it maps the rays associated to $D$ onto a set of rays associated to a dense linear manifold $D^{\prime}$ of $H$. The necessity of working with dense manifold is forced by physical interesting examples in which symmetry operators in indefinite metric spaces are described by unbounded operators. A very important example is provided by Gupta formulation of quantum electrodynamics in which Lorentz transformations are described by unbounded operators. The operators described in the previous sections clearly induce symmetry operators. That any symmetry can be regarded as induced by an operator of that kind is the result of the following theorem, which is the analogue of Wigner's theorem, in indefinite metric spaces.

Theorem. Let $T$ be a symmetry operation, i.e. an application defined on a set $\underline{D}$ of rays of an indefinite metric space $H$ onto a set $\underline{D}^{\prime}$ of rays of $H$ such that

i) the set of vectors belonging to the rays of $\underline{D}$ is a linear manifold $D$, which is dense in $H$;

ii) the set of vectors belonging to the rays of $\underline{D}^{\prime}$ is a linear manifold $D^{\prime}$, which is dense in $H$;

iii) $T$ is one to one from $\underline{D}$ to $\underline{D}^{\prime}$;

iv) $\forall \underline{x}, \underline{y} \in \underline{D}$

then

$$
|\langle T \underline{x}, T \underline{y}\rangle|=|\langle\underline{x}, \underline{y}\rangle|
$$

a) there exists an operator $U$, such that $\forall \underline{x} \in \underline{D}$, the vector $U x$ belongs to $T \underline{x}$.

b) $U$ is either $\eta$-unitary or $\eta$-antiunitary or $\eta$-pseudounitary or $\eta$-pseudoantiunitary. Clearly the last two possibilities can occur only if the eigenvalues +1 and -1 of the metric operator $\eta$ have the same multiplicity.

Remarks 1. The assumption that $D$ is linear and dense may be justified by physical considerations on symmetry operations. The condition that $D^{\prime}$ is a dense linear manifold requires some comment since one might hope to prove the linearity of $D^{\prime}$ as a consequence of the linearity of $D$ and as a byproduct of the existence of $U$. This is however not possible: see at the end of the sketch of the proof and Ref. [26].

2. It has already been mentioned that an indefinite metric space (according to Definition 1), can be reduced to a space with a metric $\eta$ satisfying $\eta^{2}=1$, by a suitable transformation. It is important to note that this can be done without spoiling the assumptions of the theorem. Namely, if $T$ is a symmetry operation in an indefinite metric space $H$ with $\eta$ bounded hermitian and $\eta^{-1}$ bounded, it is possible to introduce a new Hilbert scalar product $[\cdot, \cdot]$ and a new metric operator $\tilde{\eta}$ such that $\tilde{\eta}^{2}=1,\langle\cdot, \cdot\rangle=[\cdot, \tilde{\eta} \cdot]$ and all the assumptions of the theorem still hold. 
Before closing this section we will sketch the main lines of the proof of the theorem. A complete proof will be given in the following section. An exposition with all the technical details can be found in Ref. [26].

One of the basic tools we will use is a set of vectors $\left\{e_{i}\right\}, e_{i} \in D i=1,-1,2$, $-2, \ldots$, which have the following properties
A) $\left(e_{i}, \eta e_{j}\right)=\left\langle e_{i}, e_{j}\right\rangle=\operatorname{sign}(i) \delta_{i j}$.
B) $\left\langle z, e_{i}\right\rangle=0, \forall e_{i} \Rightarrow z=0$.

They are the analogue in $D$ of the orthonormal complete set which plays a fundamental rôle in the usual version of Wigner's theorem $(\eta=1 D=H)$. The existence of the vectors $\left\{e_{i}\right\}$ is far from obvious in the present case since in general the vectors of the subspaces $H^{ \pm}=\frac{1}{2}(1 \pm \eta) H$ do not belong to $D$.

Moreover the vectors $e_{i}$ will be found in such a way that also the set $\left\{e_{i}^{\prime}, e_{i}^{\prime} \in T \underline{e}_{i}\right\}$ is a "complete orthonormal set with respect to $\eta$ ", i.e. they satisfy
A) $\left\langle e_{n}^{\prime}, e_{m}^{\prime}\right\rangle=\lambda \delta_{n m}$,
B) $\left\langle z, e_{n}^{\prime}\right\rangle=0, \quad \forall e_{n}^{\prime}, \quad \Rightarrow z=0$.

Another basic step of the proof is that every ray associated to a finite linear combination of the $e_{i}$ 's is transformed into a ray associated to a finite linear combination of the corresponding vectors $e_{i}^{\prime}$. The construction of the operator $U$ is first given for special finite linear combinations of the $e_{i}$ 's and then extended to the generic vector of $D$, as in the proof of Wigner's theorem, in Ref. [3]. Finally $U$ is shown to be either $\eta$-unitary or $\eta$-antiunitary or $\eta$-pseudounitary or $\eta$-pseudoantiunitary. A crucial and difficult point in the proof of the theorem is the extension of $U$ from $D_{0} \equiv$ ggenerated by finite linear combinations of the $e_{i}$ 's $\}$ to $D$ since $U$ is unbounded. In general if $D_{0} \ni x_{n} \rightarrow x \in D$, as $n \rightarrow \infty, U x_{n}$ will not converge to a limit in $H$. To define $U$ on any vector of $D$ we shall first define $U$ on a suitable sequence $x_{n} \in D_{0}, n=1,-2,2,-2, \ldots$ and show that at each step the definition of $U$ on $x_{m}$ is consistent with the definition of $U$ on $x_{l},|l|<|m|$.

One might think that the linearity of $D^{\prime}$ is an unnecessary assumption if one restricts the theorem to the proof of the existence of $U$ on $D_{0}$. A counterexample shows that this is not possible [26].

\section{Proof of the Theorem}

In the proof we will assume that both $\mathrm{H}^{+}$and $\mathrm{H}^{-}$have infinite dimensions. If this is not the case the required modifications are straightforward.

Lemma 1. $0 \in D$ and $T \underline{0}=\underline{0}$.

Proof. $0 \in D$ since $D$ is a linear manifold. Furthermore since $T^{-1}$ is assumed to exist, $\forall \underline{x} \in \underline{D}^{\prime}$ one has

$$
|\langle T \underline{0}, \underline{x}\rangle|=\left|\left\langle\underline{0}, T^{-1} \underline{x}\right\rangle\right|=0 .
$$

Since $D^{\prime}$ is dense in $H,|\langle T \underline{0}, \underline{x}\rangle|=0, \forall \underline{x} \in H$, and

$$
T \underline{0}=\underline{0} \text {. }
$$


Lemma 2. There exist a set of vectors $\left\{e_{i}\right\}, i=I-\{0\}$, such that
A) $\left\langle e_{i}, e_{j}\right\rangle=\operatorname{sign}(i) \delta_{i j}$.
B) $\left\langle z, e_{i}\right\rangle=0, \forall i, \Rightarrow z=0$.
C) $e_{i} \in D, \forall i$.

Proof. The vectors $e_{i}$ are found by induction starting from an orthonormal basis of eigenvectors of $\eta:\left\{x_{i}, \eta x_{i}=\operatorname{sign}(i) x_{i} i=1,-1,2,-2 \ldots\right\}$. Let $\left\{\varepsilon_{i}\right\}$ be a sequence of positive numbers such that $\Sigma_{-\infty}^{\infty} \varepsilon_{i}^{2}<\frac{1}{16}$ and let $\left\{f_{i}\right\}$ be a set of vectors of $D$ such that $\left\|x_{i}-f_{i}\right\|<\varepsilon_{i}$, then one defines

$$
e_{1} \equiv\left(f_{1}, \eta f_{1}\right)^{-\frac{1}{2}} f_{1},
$$

where $\left(f_{1}, \eta f_{1}\right)$ is positive since $\varepsilon_{1}<\frac{1}{4}$. The induction is performed by ordering $I-\{0\}$ in the following way: $1,-1,2,-2 \ldots$ and by putting

$$
\begin{aligned}
e_{n} & \equiv \alpha_{n}\left(f_{n}-\sum_{1 \leqq i \leqq n-1}\left(e_{i}, \eta f_{n}\right) e_{i}+\sum_{-n+1 \leqq i \leqq-1}\left(e_{i}, \eta f_{n}\right) e_{i}\right) \\
& \equiv \alpha_{n} \tilde{f}_{n}, \quad \alpha_{n} \equiv\left(\tilde{f}_{n}, \eta \tilde{f}_{n}\right)^{-\frac{1}{2}}, \quad n>0 \\
e_{n} & \equiv \alpha_{n}\left(f_{n}-\sum_{1 \leqq i \leqq-n}\left(e_{i}, \eta f_{n}\right) e_{i}+\sum_{n+1 \leqq i \leqq-1}\left(e_{i}, \eta f_{n}\right) e_{i}\right), \\
& \equiv \alpha_{n} \bar{f}_{n}, \quad \alpha_{n} \equiv\left(-\bar{f}_{n}, \eta \bar{f}_{n}\right)^{-\frac{1}{2}}, \quad n \leqq 0 .
\end{aligned}
$$

The coefficients $\alpha_{n}$ are well defined since the scalar products in Eq. (14) and (15) can be shown to be strictly positive. The $e_{n}$ 's so defined clearly satisfy C). Property A) and B) can be proved by induction on $e_{k}, k$ less than $n$ in the sense of the above ordering [26].

Lemma 3. $\underline{z} \in \underline{D}^{\prime},\left|\left\langle\underline{z}, T \underline{e}_{n}\right\rangle\right|=0, \forall n, \Rightarrow \underline{z}=0$.

Proof. $0=\left|\left\langle\underline{z}, T \underline{e}_{n}\right\rangle\right|=\left|\left\langle T^{-1} \underline{z}, \underline{e}_{n}\right\rangle\right|, \forall n \Rightarrow T^{-1} \underline{z}=\underline{0} \Rightarrow \underline{z}=\underline{0}$.

Lemma 4. Let $\left\{e_{n}^{\prime}\right\}$ be a chosen sequence of vectors $e_{n}^{\prime} \in T \underline{e}_{n}$, then for any $z=\Sigma_{n \in K} a_{n} e_{n}$, with $K$ a finite set, there exists a finite sequence $a_{n}^{\prime},\left|a_{n}^{\prime}\right|=\left|a_{n}\right|$, such that $z^{\prime}=\Sigma_{n} a_{n}^{\prime} e_{n}^{\prime}$ belongs to $T \underline{z}$.

Proof. Let $z^{\prime}$ be a vector belonging to $T \underline{z}$ and $a_{n}^{\prime} \equiv\left\langle e_{n}^{\prime}, z^{\prime}\right\rangle /\left\langle e_{n}^{\prime}, e_{n}^{\prime}\right\rangle$. Because of Eq. (8), $a_{n}^{\prime} \neq 0$ only if $n \in K$. Since $D^{\prime}$ is a linear manifold $\Sigma_{n} a_{n}^{\prime} e_{n}^{\prime}$ and $z^{\prime}-\Sigma_{n} a_{n}^{\prime} e_{n}^{\prime}$ belong to $D^{\prime}$ and $\left\langle z^{\prime}-\Sigma_{n} a_{n}^{\prime} e_{n}^{\prime}, e_{k}^{\prime}\right\rangle=0, \forall k$ i.e. $z^{\prime}=\Sigma_{n \in K} a_{n}^{\prime} e_{n}^{\prime}$ and $\left|a_{n}^{\prime}\right|=\left|a_{n}\right|$ by Eq. (8).

Lemma 5. For any set of vectors $e_{n}^{\prime} \in T \underline{e}_{n}$, one has

$$
\left\langle e_{n}^{\prime}, e_{m}^{\prime}\right\rangle=\alpha\left\langle e_{n}, e_{m}\right\rangle,
$$

with $\alpha^{2}=1, \alpha$ independent of $n$ and $m$.

Proof. For a generic vector $x=\Sigma_{i \in K} a_{i} e_{i}, K$ a finite set, $x^{\prime} \in T \underline{x}$, one has by Lemma 4

$$
\begin{aligned}
\left.|\Sigma| a_{i}\right|^{2}\left\langle e_{i}, e_{i}\right\rangle \mid & =|\langle x, x\rangle|=\left|\left\langle x^{\prime}, x^{\prime}\right\rangle\right| \\
& =\left.|\Sigma| a_{i}^{\prime}\right|^{2}\left\langle e_{i}^{\prime}, e_{i}^{\prime}\right\rangle \mid .
\end{aligned}
$$

Since this equation must hold for any choice of the $a_{i}$ 's, it follows $\left\langle e_{i}^{\prime}, e_{i}^{\prime}\right\rangle=\alpha\left\langle e_{i}, e_{i}\right\rangle$, with $\alpha^{2}=1$, by the hermiticity of $\eta$. For $n \neq m$ Eq. (16) is trivially satisfied since both sides vanish. 
After these preliminary Lemmas we may now pass to the construction of the operator $U$. As a first step we have to fix the sequence of vectors $e_{n}^{\prime} \in T \underline{e}_{n}$. The prescription is given in the following Lemma, in terms of a suitable sequence of vectors.

Lemma 6. Let $\left\{z_{n}\right\}$ be a chosen sequence of vectors such that

$$
\begin{aligned}
& z_{n}=\sum_{2^{n-1} \leqq k \leqq 2^{n}} b_{k} e_{k}, \quad n>0, \\
& z_{n}=\sum_{-2^{n} \leqq k \leqq-2^{n-1}} b_{k} e_{k}, \quad n<0, \\
& z_{0}=b_{1} e_{1}+b_{-1} e_{-1}, \quad n=0,
\end{aligned}
$$

with $b_{k} \operatorname{sign} k>0$. Then one may choose $e_{n}^{\prime} \in T \underline{e}_{n}$ such that

$$
z_{n}^{\prime}=\Sigma b_{k} e_{k}^{\prime} \in T \underline{z}_{n}, \forall n \text {. }
$$

Such a choice of the $e_{n}^{\prime}$ will be denoted by $U e_{n}$, and $z_{n}^{\prime}$ by $U z_{n}$.

Proof. $e_{1}^{\prime} \in T \underline{e}_{1}$ is chosen arbitrarily and the phase of $z_{0}^{\prime}$ and $e_{-1}^{\prime} \in T \underline{e}_{-1}$ are chosen in such a way that $z_{0}^{\prime}=b_{1} e_{1}^{\prime}+b_{-1} e_{-1}^{\prime}$. By induction, when passing from $z_{N}$ to $z_{N+1}$, the phase of $z_{N+1}^{\prime}$ is chosen so that $b_{2^{N}}^{\prime}=b_{2^{N}}$, the phase of $e_{2^{N}}^{\prime}$ being already fixed, and $e_{k}^{\prime}, k>2^{N}$, is chosen in such a way that $b_{k}^{\prime}=b_{k}$.

The next step in the construction of $U$ is the extension of the vector mapping from the $z_{n}$ 's to arbitrary vectors. This is done through the following Lemmas.

Lemma 7. Let $\left\{\bar{z}_{n}\right\}$ be a sequence of vectors defined by

$$
\bar{z}_{n} \equiv \sum_{|i| \leqq 2^{n}} b_{i} \operatorname{sign}(i) e_{i}
$$

where $b_{i}$ are the coefficients entering in the definition of the $z_{n}$ 's. Then $\bar{z}_{n}^{\prime}=\Sigma_{|i| \leqq 2^{n}}$ - $b_{i} \operatorname{sign}(i) e_{i}^{\prime} \equiv U \bar{z}_{n} \in T \bar{z}_{n}$ and for any two vectors $x, y$ of the set $\left\{e_{i}\right\} \cup\left\{z_{n}\right\} \cup\left\{\bar{z}_{n}\right\}$

$$
\langle x, y\rangle=\alpha\left\langle x^{\prime}, y^{\prime}\right\rangle \equiv \alpha\langle U x, U y\rangle .
$$

Proof. By Lemma 4, $\bar{z}_{n}^{\prime}=\Sigma c_{i} e_{i}^{\prime}$, with $\left|c_{i}\right|=\left|b_{i}\right|$.

By choosing the phase of $\bar{z}_{n}^{\prime}$ such that $c_{1}=b_{1}$ and by using Eq. (8) one gets $c_{i}=b_{i} \operatorname{sign}(i)$.

Lemma 8. Let $x \in D$, with $\left\langle e_{i}, x\right\rangle \geqq 0, \forall i$, and let $x^{\prime} \equiv U x$ be chosen in the ray $T \underline{x}$ in such a way that $\alpha\left\langle e_{j}^{\prime}, x^{\prime}\right\rangle>0$, where $j$ is the index nearest to 1 (in the ordering of Lemma 2), for which $\left\langle e_{j}, x\right\rangle \neq 0$. Then, one has

$$
\left\langle e_{i}^{\prime}, x^{\prime}\right\rangle=\alpha\left\langle e_{i}, x\right\rangle, \forall i \text {. }
$$

Proof. It suffices to exploit Eq. (8) for the vectors $x$ and $\bar{z}_{n}$.

Lemma 9. Let $y \in D$ such that $\left\langle e_{k}, y\right\rangle>0$ for at least one $k$. Then $\exists y^{\prime} \equiv U y \in T \underline{y}$, such that either

or

$$
\left\langle U e_{i}, U y\right\rangle=\alpha\left\langle e_{i}, y\right\rangle, \forall i
$$

$$
\left\langle U e_{i}, U y\right\rangle=\alpha \overline{\left\langle e_{i}, y\right\rangle}, \forall i \text {. }
$$


Proof. Putting $\left\langle e_{i}, y\right\rangle \equiv c_{i} \exp \left(i \phi_{i}\right), c_{i} \geqq 0,-\pi \leqq \phi \leqq \pi$, and denoting by $k_{0}$ the index nearest to 1 such that $c_{k_{0}} \neq 0, \phi_{k_{0}}=0$, one chooses $y^{\prime} \equiv U y \in T y$ in such a way that $\left\langle e_{k_{0}}^{\prime}, y^{\prime}\right\rangle=\alpha c_{k_{0}}$ and defines $\phi_{i}^{\prime}$ so that

$$
\left\langle e_{i}^{\prime}, y^{\prime}\right\rangle=\alpha c_{i} \exp \left(i \phi_{i}^{\prime}\right) \text {. }
$$

Then, for any vector $x=\Sigma_{i \in K} d_{i} e_{i}, K$ a finite set, $d_{i} \operatorname{sign}(i)>0,\left(x^{\prime} \equiv U x\right.$ being already defined in Lemma 8), Eq. (8) for $x$ and $y$ gives

$$
\Sigma d_{i} d_{j}\left[\exp i\left(\phi_{i}-\phi_{j}\right)\right] c_{i} c_{j}=\Sigma d_{i} d_{j} c_{i} c_{j} \exp \left[i\left(\phi_{i}^{\prime}-\phi_{j}^{\prime}\right)\right]
$$

i.e. either $\phi_{i}^{\prime}=\phi_{i}, \forall i$, or $\phi_{i}^{\prime}=-\phi_{i}, \forall i$.

Lemma 10. $\forall z \in D, \exists z^{\prime} \equiv U z \in T \underline{z}$ such that either

I) $\left\langle e_{i}^{\prime}, z^{\prime}\right\rangle=\alpha\left\langle e_{i}, z\right\rangle, \forall i$

or

II) $\left\langle e_{i}^{\prime}, z^{\prime}\right\rangle=\alpha\left\langle z, e_{i}\right\rangle, \forall i$.

Furthermore the same equation holds for all the vectors of $D$.

Proof. Given $z$, one considers the vector $\tilde{z}=\exp \left(-i \phi_{k_{0}}\right) z$, where $k_{0}$ is the index nearest to one such that $\left\langle e_{k_{0}}, z\right\rangle=c_{k_{0}} \exp \left(i \phi_{k_{0}}\right), c_{k_{0}}>0 . \tilde{z}^{\prime}=U \tilde{z}$ is then defined as in Lemma 9 and one chooses

$$
\begin{aligned}
& z^{\prime} \equiv U z=\exp \left(i \phi_{k_{0}}\right) \tilde{z}^{\prime}, \quad \text { if } \quad\left\langle e_{i}^{\prime}, \tilde{z}^{\prime}\right\rangle=\alpha\left\langle e_{i}, \tilde{z}\right\rangle \\
& z^{\prime} \equiv U z=\exp \left(-i \phi_{k_{0}}\right) \tilde{z}^{\prime}, \quad \text { if } \quad\left\langle e_{i}^{\prime}, \tilde{z}^{\prime}\right\rangle=\alpha\left\langle\tilde{z}, e_{i}\right\rangle .
\end{aligned}
$$

The proof that in the first case Eq. (26) holds for all the vectors of $D$ and in the second case Eq. (27) is valid for all the vectors of $D$ essentially follows the argument given in Ref. [3] for the standard Wigner's theorem (see Appendix).

Lemma 11. The mapping $z \rightarrow U z, \forall z \in D$, defined in Lemma 10 is linear in the Case I and antilinear in the Case II. Moreover, in Case I, for any two vectors $x, y \in D$, one has

and in Case II

$$
\langle x, y\rangle=\alpha\langle U x, U y\rangle
$$

$$
\langle x, y\rangle=\alpha\langle U y, U x\rangle .
$$

Proof. Case I. $\forall x, y \in D$ one has, $\forall i$

$$
\left\langle e_{i}^{\prime}, U(a x+b y)\right\rangle=\alpha\left\langle e_{i}, a x+b y\right\rangle=a\left\langle e_{i}^{\prime}, U x\right\rangle+b\left\langle e_{i}^{\prime}, U y\right\rangle
$$

and linearity follows from Lemma 3 . To prove Eq. (28), $\forall x, y \in D,\langle x, y\rangle \neq 0$, one puts $\langle U x, U y\rangle=\exp [$ if $(x, y)]\langle x, y\rangle$. Then for every vector $z \in D$ such that $\langle x, z\rangle \neq 0$

$$
\begin{aligned}
\langle x, y+b z\rangle \exp [\text { if }(x, y+b z)]= & \exp [\text { if }(x, y)]\langle x, y\rangle \\
& +b \exp [\text { if }(x, z)]\langle x, z\rangle .
\end{aligned}
$$

For $b=\langle x, y\rangle /\langle x, z\rangle$, the above equation implies $f(x, y)=f(x, z)$. Putting $z=e_{i}$, one obtains $\exp [$ if $(x, y)]=\alpha$.

This concludes the proof of the theorem. 


\section{Appendix}

Completion of the proof of Lemma 10. Suppose that $x, y \in D$ such that $\left\langle e_{i}^{\prime}, x^{\prime}\right\rangle$ $=\alpha\left\langle e_{i}, x\right\rangle \equiv \alpha c_{i} e^{i \phi_{i}},\left\langle e_{i}^{\prime}, y^{\prime}\right\rangle=\alpha\left\langle y, e_{i}\right\rangle \equiv \alpha d_{i} e^{i \psi_{i}}$ and that not all the phases $\phi_{i}$ (and $\psi_{i}$ ) differ by multiples of $\pi$ [otherwise Eq. (26) and (27) may be reduced one to the other by an overall change of phase in the definition of $x^{\prime}$ or $\left.y^{\prime}\right]$.

Then one can find a continuous curve $x(t), t \in[0,1], x(t) \in D$, with $x(0)=x$, $x(1)=y$, such that $\forall t \in[0,1]$, the phases of $x(t)$ are not all differing by multiples of $\pi$, e.g.

$$
x(t)=t y-(1-t) x+\sum_{i \in K} a_{i}(t) e_{i},
$$

$K$ a finite set, $a_{i}(t)$ continuous functions on $[0,1], a_{i}(0)=a_{i}(1)=0$. By the properties of the extremes of the curve, there must exist a $\bar{t} \in[0,1]$ such that there is a sequence $x\left(t_{n}\right) \rightarrow x(\bar{t})$ with the property that either

or

$$
\begin{aligned}
& \left\langle e_{i}^{\prime}, x^{\prime}\left(t_{n}\right)\right\rangle=\alpha\left\langle e_{i}, x\left(t_{n}\right)\right\rangle,\left\langle e_{i}^{\prime}, x^{\prime}(\bar{t})\right\rangle=\alpha \overline{\left\langle e_{i}, x(\bar{t})\right\rangle} \\
& \left\langle e_{i}^{\prime}, x^{\prime}\left(t_{n}\right)\right\rangle=\alpha\left\langle x\left(t_{n}\right), e_{i}\right\rangle,\left\langle e_{i}^{\prime}, x^{\prime}(\bar{t})\right\rangle=\alpha \overline{\left\langle x(\bar{t}), e_{i}\right\rangle} .
\end{aligned}
$$

Suppose that case (A1) occurs. Then one shows that this leads to a contradiction. [The proof for the case (A2) is analogous.] To this purpose, let $i, j$ be two indices such that the phases of $\left\langle e_{i}, x(\bar{t})\right\rangle$ and $\left\langle e_{j}, x(\bar{t})\right\rangle$ do not differ by multiples of $\pi$. Putting $z_{i j}(s) \equiv e_{i}+s e_{j}$ one obtains $z_{i j}(s)^{\prime}=e_{i}^{\prime}+s^{\prime} e_{j}^{\prime}$ with $s^{\prime}=\bar{s}, \forall s \in C\left[s^{\prime}=s, \forall s\right.$ if case (A2) occurs]. In fact, $\forall s \in C$, using (A1)

$$
\begin{aligned}
\left|\left\langle e_{i}, x(\bar{t})\right\rangle+\bar{s}\left\langle e_{j}, x(\bar{t})\right\rangle\right| & =\left|\left\langle z_{i j}(s), x(\bar{t})\right\rangle\right| \\
& =\mid\left\langle e_{i}^{\prime}, x^{\prime}(\bar{t})\right\rangle+\bar{s}^{\prime}\left\langle e_{j}^{\prime}, x^{\prime}(\bar{t})\right\rangle \\
& =\left|\left\langle e_{i}, x(\bar{t})\right\rangle+s^{\prime}\left\langle e_{j}, x(\bar{t})\right\rangle\right| .
\end{aligned}
$$

This is possible only if $s^{\prime}=\bar{s}, \forall s \in C$.

To find the contradiction one computes $\lim _{n \rightarrow \infty}\left\langle z_{i j}(s), x\left(t_{n}\right)\right\rangle \mid$ in two different ways. First

secondly

$$
\begin{aligned}
\lim _{n \rightarrow \infty}\left|\left\langle z_{i j}(s), x\left(t_{n}\right)\right\rangle\right| & =\left|\left\langle z_{i j}(s), x(\bar{t})\right\rangle\right| \\
& =\left|\left\langle e_{i}, x(\bar{t})\right\rangle+\bar{s}\left\langle e_{j}, x(\bar{t})\right\rangle\right|
\end{aligned}
$$

$$
\begin{aligned}
\lim _{n \rightarrow \infty}\left|\left\langle z_{i j}(s), x\left(t_{n}\right)\right\rangle\right| & =\lim _{n \rightarrow \infty}\left|\left\langle z_{i j}(s)^{\prime}, x^{\prime}\left(t_{n}\right)\right\rangle\right| \\
& =\lim _{n \rightarrow \infty}\left|\left\langle e_{i}, x\left(t_{n}\right)\right\rangle+s\left\langle e_{j}, x\left(t_{n}\right)\right\rangle\right| \\
& =\left|\left\langle e_{i}, x(\bar{t})\right\rangle+s\left\langle e_{j}, x(\bar{t})\right\rangle\right| .
\end{aligned}
$$

Acknowledgements. One of us (F.S.) would like to thank Prof. V. Bargmann for a fruitful discussion.

\section{References}

1. Wigner,E.P.: Group theory and its application to the quantum, mechanics of atomic spectra. New York: Academic Press 1959

2. Bargmann, V.: Journ. Math. Phys. 5, 862 (1964)

3. Bargmann, V., Wightman, A.S., Wigner, E.P.: Unpublished manuscript (1959)

4. Wightman, A.S.: Nuovo Cimento Suppl. XIV, 81 (1959)

5. Ulhorn, U.: Arkiv Fysik 23, 307 (1963) 
6. Birkhoff, G., Neumann, J.Von: Ann. Math. 37, 823 (1936)

7. Baer, R.: Linear algebra and projective geometry. New York: Academic Press 1952

8. Dirac, P.A. M.: Proc. Roy. Soc. A 180, 1 (1942)

Pauli, W.: Rev. Mod. Phys. 15, 175 (1943)

Heisenberg, W.: Introduction to the unified field theory of elementary particles. London: Wiley and Sons 1966

9. Lee, T. D.: Phys. Rev. 95, 1329 (1954)

10. Källen, G., Pauli, W.: Kgl. Danske Videnskab. Selskab Mat. Fys. Medd. 30, 23 (1955)

11. Lee, T.D., Wick, G.C.: Nuclear Physics B9, 209 (1969); Nuclear Physics B 10, 1 (1969); Phys. Rev. D2, 1033 (1970); Phys. Rev. D3, 1046 (1971)

12. Sudarshan, E.C. G.: Phys. Rev. 123, 2183 (1961)

13. Lee, T.D., Yang, C. N.: Phys. Rev. 128, 885 (1962)

14. Hooft, G.'t: Nuclear Physics B33, 173 (1971); Nuclear Physics B35, 167 (1971)

15. Weinberg, S.: Phys. Rev. Letters 27, 1688 (1971)

16. Hooft, G.t', Veltman, M.: Nuclear Physics B 44, 189 (1972)

17. Wightman, A.S., Gårding,L.: Arkiv Fysik 28, 129 (1964);

Strocchi, F.: Phys. Rev. 162, 1429 (1967); Phys. Rev. D2, 2334 (1970)

18. Ferrari, R., Picasso, L.E., Strocchi, F.: Commun. math. Phys. 35, 25 (1974)

19. Strocchi, F., Wightman, A.S.: Proof of the charge superselection rule in local relativistic quantum field theory (to be published, Section 4)

20. Pontryiagin, L.S.: Izv. Akad. Nauk. SSSR Ser. Mat. 8, 243 (1944)

21. Ginzburg, Yu.P., Iohvidov, I. S.: Russian Mathematical Surveys, 17, No. 4, 1 (1962)

22. Krein, M. G.: Second mathematical summer school. Part I. Naukoav Dumka Kiev (1965)

23. Krein, M.G., Langer, H.: Dokl. Akad. Nauk SSSR 152, 1053 (1964); ibid. SSSR 154, 1258 (1964) Krein,M.G., Langer,H.: Proc. Internat. Sympos. Appl. Theory of Functions in Continuum Mechanics (Tbilisi, 1963). Vol. II: Fluid and gas mechanics. Math. Methods, „Nauka”, Moscow 1965 , p. 283

Krein, S. G.: Dokl. Akad. Nauk SSSR, 159, 262 (1964)

24. Krein, M. G., Ljubarskii, G. Ja.: Izv. Akad. Nauk SSSR Ser. Mat. 26, 549 (1962)

Kucer, D. L.: Naucn. Zap. Kaf. Mat. Fiz. Estest. Odessa Gos. Ped. Inst. 25 (2), 25 (1961)

Kühne, R.: Dissertation, Dresden (1964)

Lapter, G.I.: Dissertation, Voronezh (1964)

25. Krein, M.G., Jakubovic, V.A.: Proc. Internat. Sympos. Non-Linear Vibrations (1961), Vol. 1, p. 277. Izdat. Akad. Nauk Ukrain. SSR Kiev 1963

26. Morchio, G.: Thesis, SNS, Pisa; Internal Report SNS, Pisa, April 1974

Communicated by H. Arakı

\author{
L. Bracci \\ G. Morchio \\ F. Strocchi \\ Scuola Normale Superiore \\ Piazza dei Cavalieri \\ I-56100 Pisa, Italy
}


